\title{
ACCELERATION SCHEMES OF THE DISCRETE VELOCITY METHOD: GASEOUS FLOWS IN RECTANGULAR MICROCHANNELS*
}

\author{
D. VALOUGEORGIS ${ }^{\dagger}$ AND S. NARIS ${ }^{\dagger}$
}

\begin{abstract}
The convergence rate of the discrete velocity method (DVM), which has been applied extensively in the area of rarefied gas dynamics, is studied via a Fourier stability analysis. The spectral radius of the continuum form of the iteration map is found to be equal to one, which justifies the slow convergence rate of the method. Next the efficiency of the DVM is improved by introducing various acceleration schemes. The new synthetic-type schemes speed up significantly the iterative convergence rate. The spectral radius of the acceleration schemes is also studied and the so-called $\mathrm{H}_{1}$ acceleration method is found to be the optimum one. Finally, the two-dimensional flow problem of a gas through a rectangular microchannel is solved using the new fast iterative DVM. The number of required iterations and the overall computational time are significantly reduced, providing experimental evidence of the analytic formulation. The whole approach is demonstrated using the BGK and S kinetic models.
\end{abstract}

Key words. iterative methods, acceleration schemes, rarefied gas flows

AMS subject classifications. 65B99, 76P05

PII. S1064827502406506

1. Introduction. After the early work of Broadwell [4], Huang et al. [10], and Cabannes [6], the discrete velocity method (DVM) has been developed into one of the most common techniques for solving the Boltzmann equation [7, 12] and simplified model equations $[14,23,15]$ in the area of rarefied gas dynamics. The method has also been applied to solve mixture problems $[20,8]$. An extensive review article on internal rarefied gas flows including DVM applications has been given lately by Sharipov [16]. Very recently, new models of discrete velocity gases [5] and mixtures [9] have been introduced indicating that the method can be extended into more general models including polyatomic gases with chemical reactions.

The method is based on a discretization of the velocity and space variables by choosing a suitable set of discrete velocities and by applying a consistent finite difference scheme, respectively. Then the collision integral term is approximated by an appropriate quadrature, and the resulting discrete system of equations is solved in an iterative manner. Researchers implementing the DVM are well aware, however, of its slow convergence, particularly when domains with thick subregions are considered [17]. In these cases a large number of iterations are required, and calculations are amenable to accumulated round-off error. Special attention is needed to sustain acceptable accuracy. Even more when multidimensional physical systems are examined, computational effort and time are drastically increased. These types of calculations are now needed to solve in an efficient and accurate manner fluidics applications in micro-electrical-mechanical systems and nanotechnology problems. In these applications, due to the fact that the Navier-Stokes equations are restricted by the hydrodynamic regime, kinetic-type equations and corresponding numerical approaches must

\footnotetext{
${ }^{*}$ Received by the editors April 30, 2002; accepted for publication (in revised form) August 10, 2002; published electronically DATE.

http://www.siam.org/journals/sisc/x-x/40650.html

${ }^{\dagger}$ Department of Mechanical and Industrial Engineering, University of Thessaly, Pedion Areos, Volos, 38334, Greece (diva@mie.uth.gr, snaris@mie.uth.gr). The research of the second author was partially supported by Association EURATOM-Hellenic Republic contract ERB 5005 CT 990100.
} 
be introduced. Recently, the DVM has been applied to solve the isothermal [18] and nonisothermal [19] gas flow problems through rectangular microchannels.

The renewed increased interest for large-scale computational solutions based on the DVM makes it necessary to study carefully its convergence rate and propose novel accelerated iterative schemes. This type of fast iterative algorithms has been well developed in the area of neutron transport $[11,1,2]$. They have been applied effectively to speed up the iterative convergence of the discrete-ordinates method in optically thick regions with low absorption and isotropic or anisotropic scattering [21]. As it is well known, the areas of neutron transport and kinetic theory are described by similar-type linear integro-differential equations derived from the Boltzmann equation, while the discrete ordinates and velocity methods are based on common principals and they have similar characteristics and behavior. Thus it is reasonable to expect that the acceleration schemes, which have been well developed for the discrete-ordinates method, can be extended with certain modifications to speed up the convergence rate of the DVM. In the present work we extend these ideas in the area of rarefied gas dynamics, and we develop suitable new accelerated algorithms of the classical DVM for the solution of the well-known BGK [3] and S [13] model kinetic equations [16].

We begin our analysis in section 2 with the stability Fourier analysis of the typical DVM, and the spectral radius of the method is found for the BGK and S model kinetic equations. We continue in section 3 with the formulation of the proposed acceleration schemes for both kinetic models, while in section 4 results of the theoretical convergence rates of certain acceleration methods are presented for the BGK model. In section 5 we perform a numerical experiment, solving the two-dimensional flow problem of a gas through a rectangular microchannel due to a pressure and temperature gradient, and the performance of the accelerated schemes is demonstrated. Finally, in section 5 a brief summary and some concluding remarks are given.

It is noticed that our theoretical solutions of the convergence rates in sections 2 and 4 are based on the analytic transport and acceleration equations with no discretization in velocity and space. Furthermore, the use of the infinite medium problem for the Fourier analysis is highly idealized. However, previous experience with acceleration schemes $[1,21]$ has shown that these analytical results are indicative for the associated discretized versions. This statement is shown to be true in section 5 , where the fully discretized schemes are implemented to solve the two-dimensional flow problem with Dirichlet boundary conditions.

2. Stability analysis of the DVM. In many cases, due to the complexity of the collision term of the Boltzman equation, certain simplified kinetic models are implemented. Two of the most commonly tested models are the BGK and the S models, which have been applied to solve a large number of rarefied gas dynamics problems based on the DVM [16]. Thus we base our stability analysis approach on these two particular models.

For simplicity and demonstration purposes, the stability analysis approach will be presented first for the one-dimensional linearized BGK model equation for shear flow problems in slab geometry [7]. In this case the analytic version of the DVM is described by the transport equation

$$
\mu \frac{\partial \psi(x, \mu)^{(\ell+1 / 2)}}{\partial x}+\psi(x, \mu)^{(\ell+1 / 2)}=\varphi_{0}(x)^{(\ell)}+S(x)
$$

with

$$
\varphi_{0}(x)^{(\ell+1)}=\frac{1}{\sqrt{\pi}} \int_{-\infty}^{\infty} \psi(x, \mu)^{\left(\ell+\frac{1}{2}\right)} e^{-\mu^{2}} d \mu
$$


where $x \in(-\infty, \infty)$ is the spatial variable, $\mu \in(-\infty, \infty)$ is the particle velocity in the $x$ direction, $S(x)$ is a source term depending upon the problem under consideration, and $\ell$ denotes the iteration index. The term $\phi_{0}$ can be considered as the zeroth Hermite moment of $\psi$. This concept is generalized later, in section 3, where higher order Hermite moments of $\psi$ are considered. The convergence rate of the iteration map described by (1) and (2) is studied by the following Fourier analysis, which is applied to the infinite medium problem. We define the correction (or error) functions

$$
Y(x, \mu)^{(\ell+1 / 2)}=\psi(x, \mu)^{(\ell+1 / 2)}-\psi(x, \mu)^{(\ell-1 / 2)}
$$

and

$$
\Phi_{0}(x)^{(\ell+1)}=\varphi_{0}(x)^{(\ell+1)}-\varphi_{0}(x)^{(\ell)}
$$

as the difference between successive iteratives of $\psi$ and $\varphi_{0}$, respectively. The rate at which $Y(x, \mu)^{(\ell+1 / 2)}$ and $\Phi_{0}(x)^{(\ell+1)}$ tend to zero is the rate of convergence of the DVM. By subtracting (1) and (2) for successive iterates we obtain

$$
\mu \frac{\partial Y(x, \mu)^{\left(\ell+\frac{1}{2}\right)}}{\partial x}+Y(x, \mu)^{\left(\ell+\frac{1}{2}\right)}=\Phi_{0}(x)^{(\ell)}
$$

and

$$
\Phi_{0}(x)^{(\ell+1)}=\frac{1}{\sqrt{\pi}} \int_{-\infty}^{\infty} Y(x, \mu)^{\left(\ell+\frac{1}{2}\right)} e^{-\mu^{2}} d \mu .
$$

To determine the convergence rate we seek eigenvalues $\omega$ and eigenfunctions $y(\mu)$ of the form

$$
Y(x, \mu)^{(\ell+1 / 2)}=\omega^{\ell} y(\mu) e^{i \lambda x}
$$

and

$$
\Phi_{0}(x)^{(\ell+1)}=\omega \Phi_{0}(x)^{(\ell)}=\omega^{2} \Phi_{0}(x)^{(\ell-1)}=\cdots=\omega^{\ell+1} \alpha_{0} e^{i \lambda x},
$$

where $a_{0}$ is some unknown constant. In (7) and (8), $\omega$ is the eigenvalue corresponding to the Fourier wavenumber $\lambda$ and represents the error from one iteration to the next. Equations (7) and (8) are introduced into (5) and (6) to obtain expressions for the eigenfunction and the eigenvalue

$$
y(\mu)=\frac{\alpha_{0}}{1+i \lambda \mu}
$$

and

$$
\omega=\omega_{\mathrm{DVM}}=\frac{1}{\sqrt{\pi}} \int_{-\infty}^{\infty} \frac{e^{-\mu^{2}}}{1+(\lambda \mu)^{2}} d \mu=\Lambda
$$

respectively. To distinguish this result from the following ones obtained by the accelerated schemes, the eigenvalue $\omega$ of the DVM is denoted by $\omega_{\text {DVM }}$. The spectral radius $\sup _{\lambda}\left|\omega_{\mathrm{DVM}}\right|$, with $-\infty<\lambda<\infty$, represents the slowest possible reduction in the error from one iteration to the next. The integral in (10) is computed numerically, and the complete plot of $\omega$ versus $\lambda$ is given in Figure 1. The maximum value of $\left|\omega_{\text {DVM }}\right|$, which occurs for $\lambda=0$, is $\omega_{\mathrm{DVM}}=1$. For a finite system the flat $\lambda=0$ mode, 
with the corresponding eigenfunction $y(\mu)=\alpha_{0}$, cannot be present and thus the DVM described by (1) and (2), when applied to more realistic finite systems with boundary conditions, converges absolutely. At the same time, however, the slow convergence rate of the method in the case of thick slabs is well understood and justified.

Next we consider the convergence rate of the one-dimensional linearized S-model kinetic equations, which is commonly implemented when nonisothermal flows are considered in order to obtain the correct Prandtl number. The analytic iteration map of the DVM is described by the two-coupled equations

$$
\mu \frac{\partial \psi_{1}(x, \mu)^{\left(\ell+\frac{1}{2}\right)}}{\partial x}+\psi_{1}(x, \mu)^{\left(\ell+\frac{1}{2}\right)}=f(x)^{(\ell)}+\frac{2}{15}\left(\mu^{2}-\frac{1}{2}\right) g(x)^{(\ell)}+S(x)
$$

and

(11b) $\mu \frac{\partial \psi_{2}(x, \mu)^{\left(\ell+\frac{1}{2}\right)}}{\partial x}+\psi_{2}(x, \mu)^{\left(\ell+\frac{1}{2}\right)}=f(x)^{(\ell)}+\frac{2}{15}\left(\mu^{2}+\frac{1}{2}\right) g(x)^{(\ell)}+S(x)$

and the corresponding moments

$$
f(x)^{(\ell+1)}=\frac{1}{\sqrt{\pi}} \int_{-\infty}^{\infty} \psi_{1}(x, \mu)^{\left(\ell+\frac{1}{2}\right)} e^{-\mu^{2}} d \mu
$$

and

$g(x)^{(\ell+1)}=\frac{1}{\sqrt{\pi}} \int_{-\infty}^{\infty}\left(\mu^{2}-\frac{5}{2}\right) \psi_{1}(x, \mu)^{\left(\ell+\frac{1}{2}\right)} e^{-\mu^{2}} d \mu+\frac{2}{\sqrt{\pi}} \int_{-\infty}^{\infty} \psi_{2}(x, \mu)^{\left(\ell+\frac{1}{2}\right)} e^{-\mu^{2}} d \mu$.

Operating on a similar manner as before we define the correction functions

$$
\begin{aligned}
R(x, \mu)^{(\ell+1 / 2)} & =\psi_{1}(x, \mu)^{(\ell+1 / 2)}-\psi_{1}(x, \mu)^{(\ell-1 / 2)}, \\
S(x, \mu)^{(\ell+1 / 2)} & =\psi_{2}(x, \mu)^{(\ell+1 / 2)}-\psi_{2}(x, \mu)^{(\ell-1 / 2)}, \\
Z_{0}(x)^{(\ell+1)} & =f(x)^{(\ell+1)}-f(x)^{(\ell)},
\end{aligned}
$$

and

$$
W_{0}(x)^{(\ell+1)}=g(x)^{(\ell+1)}-g(x)^{(\ell)} .
$$

Then (11) is subtracted for successive iterates to yield

$$
\mu \frac{d R(x, \mu)^{\left(\ell+\frac{1}{2}\right)}}{d x}+R(x, \mu)^{\left(\ell+\frac{1}{2}\right)}=Z_{0}(x)^{(\ell)}+\frac{2}{15}\left(\mu^{2}-\frac{1}{2}\right) W_{0}(x)^{(\ell)}
$$

and

$$
\mu \frac{d S(x, \mu)^{\left(\ell+\frac{1}{2}\right)}}{d x}+S(x, \mu)^{\left(\ell+\frac{1}{2}\right)}=Z_{0}(x)^{(\ell)}+\frac{2}{15}\left(\mu^{2}+\frac{1}{2}\right) W_{0}(x)^{(\ell)}
$$

with

$$
Z_{0}(x)^{(\ell+1)}=\frac{1}{\sqrt{\pi}} \int_{-\infty}^{\infty} R(x, \mu)^{\left(\ell+\frac{1}{2}\right)} e^{-\mu^{2}} d \mu
$$

and

$$
\begin{aligned}
& W_{0}(x)^{(\ell+1)} \\
& =\frac{1}{\sqrt{\pi}} \int_{-\infty}^{\infty}\left(\mu^{2}-\frac{5}{2}\right) R(x, \mu)^{\left(\ell+\frac{1}{2}\right)} e^{-\mu^{2}} d \mu+\frac{2}{\sqrt{\pi}} \int_{-\infty}^{\infty} S(x, \mu)^{\left(\ell+\frac{1}{2}\right)} e^{-\mu^{2}} d \mu .
\end{aligned}
$$


Applying a separation of variables, Fourier mode solution for the infinite-medium model problem in the same manner as before we set

$$
\begin{aligned}
R(x, \mu)^{(\ell+1 / 2)} & =\omega^{l} r(\mu) e^{i \lambda x}, \\
S(x, \mu)^{(\ell+1 / 2)} & =\omega^{l} s(\mu) e^{i \lambda x}, \\
Z_{0}(x)^{(\ell+1)} & =\omega Z_{0}(x)^{(\ell)}=\omega^{2} Z_{0}(x)^{(\ell-1)}=\cdots=\omega^{\ell+1} z_{0} e^{i \lambda x},
\end{aligned}
$$

and

$$
W_{0}(x)^{(\ell+1)}=\omega W_{0}(x)^{(\ell)}=\omega^{2} W_{0}(x)^{(\ell-1)}=\cdots=\omega^{\ell+1} w_{0} e^{i \lambda x},
$$

where $z_{0}$ and $w_{0}$ are unknown constants. Substituting the above Fourier mode ansatz into (14), the expressions for the two eigenfunctions

$$
r(\mu)=\frac{z_{0}+\frac{2}{15}\left(\mu^{2}-\frac{1}{2}\right) w_{0}}{1+i \lambda \mu}
$$

and

$$
s(\mu)=\frac{z_{0}+\frac{2}{15}\left(\mu^{2}+\frac{1}{2}\right) w_{0}}{1+i \lambda \mu}
$$

are obtained. Introducing then (17) for $r(\mu)$ and $s(\mu)$ into (16) and the resulting equations into (15) yields the $2 \times 2$ matrix eigenvalue problem

$$
(A-\omega I) \alpha=0 .
$$

In vector equation (18), $\omega$ are the two eigenvalues of $A$ indicating the convergence rate of the DVM, $\alpha=\left[z_{0}, w_{0}\right]^{T}$ are any of the two corresponding eigenvectors, and $A$ is a $2 \times 2$ matrix given by

$$
A(\lambda)=\left[\begin{array}{cc}
\Lambda & \frac{2}{15}\left[\frac{1-\Lambda}{\lambda^{2}}-\frac{\Lambda}{2}\right] \\
{\left[\frac{1-\Lambda}{\lambda^{2}}-\frac{\Lambda}{2}\right]} & \frac{3}{10} \Lambda-\frac{2}{15} \frac{1}{\lambda^{2}}\left[\frac{1-\Lambda}{\lambda^{2}}-\Lambda+\frac{1}{2}\right]
\end{array}\right]
$$

where $\Lambda$ is the integral defined in (10). Closed form expressions for the two eigenvalues can be easily obtained by solving analytically the quadratic characteristic equation. Then the closed form expressions are computed numerically as functions of $\lambda$ and the results are plotted in Figure 1. It is found that as $\lambda$ approaches zero the values of $\omega_{1}(\lambda)$ and $\omega_{1}(\lambda)$ are increased and the maximum values of $\left|\omega_{1}\right|$ and $\left|\omega_{2}\right|$, which occur for $\lambda=0$, are $\omega_{1}=1$, and $\omega_{2}=\frac{1}{3}$. Thus the spectral radius of the DVM for the $\mathrm{S}$ model is equal to one, and it is the same with the spectral radius obtained for the BGK model. Actually as it is shown in Figure 1, the values of the whole spectrum of $\omega_{1}(\lambda)$ for the $\mathrm{S}$ model is very close to the values of $\omega(\lambda)$ for the BGK model. The second eigenvalue $\omega_{2}(\lambda)$ is less than $\omega_{1}(\lambda)$ for all $\lambda \geq 0$. Again the slow convergence rate of the DVM applied to the $S$ model kinetic equation, particularly when thick regions are considered, is explained. 


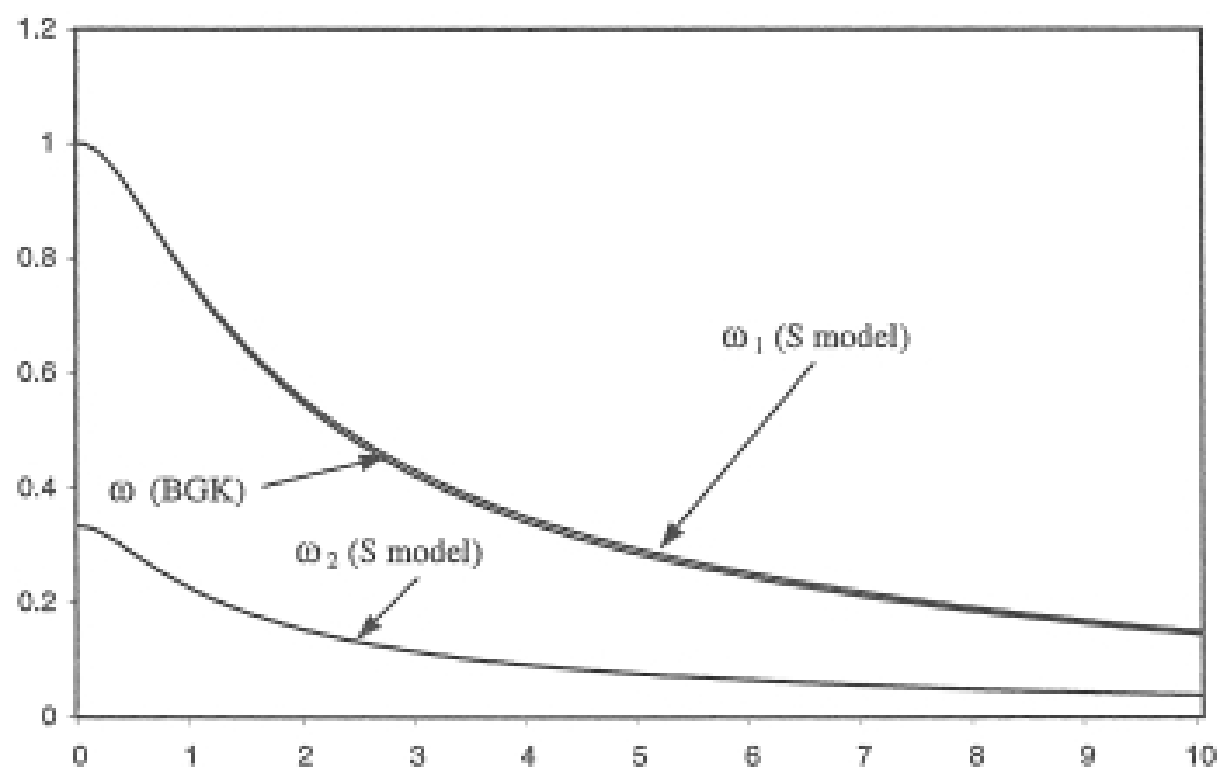

FIG. 1. Plots of $\omega$ versus $\lambda$ for the typical DVM (BGK and $S$ models).

The above Fourier mode analysis can be extended in a straightforward manner in multidimensional Cartesian geometry. For example, in a two-dimensional case the BGK equation may be written as

$$
\mu \frac{\partial \psi(x, y, \mu, \eta)}{\partial x}^{\left(\ell+\frac{1}{2}\right)}+\eta \frac{\partial \psi(x, y, \mu, \eta)}{\partial y}^{\left(\ell+\frac{1}{2}\right)}+\psi(x, y, \mu, \eta)^{\left(\ell+\frac{1}{2}\right)}=\varphi_{00}^{(l)}(x, y)+S(x, y)
$$

with

$$
\varphi_{00}^{(\ell+1)}(x, y)=\frac{1}{\pi} \int_{-\infty}^{\infty} \int_{-\infty}^{\infty} \psi^{\left(\ell+\frac{1}{2}\right)}(x, y, \mu, \eta) e^{-\mu^{2}-\eta^{2}} d \mu d \eta,
$$

where $x, y \in(-\infty, \infty)$ are the spatial variables, $\mu, \eta \in(-\infty, \infty)$ are the particle velocities in the $x$ and $y$ directions, respectively, $\psi(x, y, \mu, \eta)$ is the unknown function, $S(x, y)$ is a source term depending upon the problem under consideration, and $\ell$ denotes the iteration index. Subtracting (20) and (21) for successive iterates we find

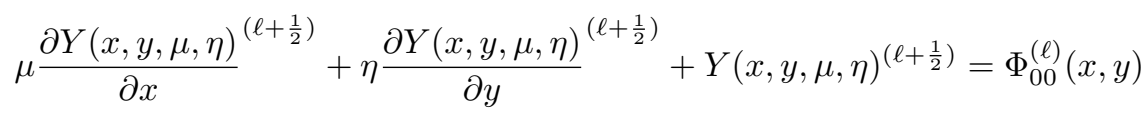

with

$$
\Phi_{00}^{(\ell+1)}(x, y)=\frac{1}{\pi} \int_{-\infty}^{\infty} \int_{-\infty}^{\infty} Y^{\left(\ell+\frac{1}{2}\right)}(x, y, \mu, \eta) e^{-\mu^{2}-\eta^{2}} d \mu d \eta
$$

where $Y(x, y, \mu, \eta)$ and $\Phi_{00}(x, y)$ are the correction functions. Substituting

$$
Y(x, y, \mu, \eta)^{(\ell+1 / 2)}=\omega^{\ell} y(\mu, \eta) e^{i \lambda\left(x \theta_{x}+y \theta_{y}\right)}
$$


and

$$
\Phi_{00}(x)^{(\ell+1)}=\omega \Phi_{00}(x)^{(\ell)}=\omega^{\ell+1} \alpha_{00} e^{i \lambda x},
$$

where $\lambda=\lambda\left(\theta_{x}, \theta_{y}\right)$, with $\theta_{x}^{2}+\theta_{y}^{2}=1$, into (22) and (23) and after some routine manipulation, the following closed form expressions are obtained for the eigenfunction:

$$
y(\mu, \eta)=\frac{\alpha_{00}}{1+i \lambda\left(\mu \theta_{x}+\eta \theta_{y}\right)}
$$

and the eigenvalue

$$
\omega(\lambda)=\frac{1}{\pi} \int_{-\infty}^{\infty} \int_{-\infty}^{\infty} \frac{e^{-\mu^{2}-\eta^{2}}}{1+\lambda^{2}\left(\mu \theta_{x}+\eta \theta_{y}\right)^{2}} d \mu d \eta .
$$

Applying a straightforward linear transformation, the double integral in (27) is reduced to the single integral in (10), which has been derived for the one-dimensional case. As it is expected the continuous plane solutions of the exact kinetic equations are reduced to the solutions of the corresponding slab geometry equations. Thus the iteration scheme applied to the exact kinetic equations must perform equally well or poorly in all Cartesian geometries. This conclusion holds also for the $\mathrm{S}$ model kinetic equations as well as for the proposed acceleration schemes. Before we proceed with the formulation of the iterative acceleration schemes, it is pointed out that transport (1), (11), and (20) coupled with the corresponding moments (2), (12), and (21), respectively, represent a wide range of classical rarefied gas dynamics problem, depending upon the types of the boundary conditions. Typical examples are the Poiseuille and thermal creep flows in slabs, cylinders, and orthogonal ducts. On the boundary conditions issue we will comment in section 5 , where numerical results are presented.

3. Formulation of the acceleration schemes for the BGK and S models. The development of fast iteration schemes for the solution of the neutron and radiative transport equations has been carried out by many researchers and has led to dramatic theoretical and practical success $[1,2,21]$. The most advanced of these acceleration methods are based on the formulation of moment equations, the so-called synthetic equations, which are solved coupled with the transport equation to improve significantly the slow iterative convergence rate of the transport equation. Here similar synthetic type equations are obtained for the BGK and S models. It is noticed that although the DVM has been applied extensively to solve kinetic theory problems in an iterative manner, no effort has been made until now to accelerate the slow convergence rate of the iteration map.

3.1. The BGK model kinetic equation. The proposed acceleration scheme is formulated based on the linearized one-dimensional BGK transport equation. Extending earlier work in the areas of neutron and radiative transport [1, 2], (1) is first multiplied by the exponential term $\frac{1}{\sqrt{\pi}} e^{-\mu^{2}}$ and then the first $N+1$ Hermite moments of the resulting equation are taken to obtain a set of ordinary differential equations

$$
\begin{aligned}
& \frac{1}{2} \frac{d \varphi_{1}(x)^{\left(\ell+\frac{1}{2}\right)}}{d x}+\varphi_{0}(x)^{\left(\ell+\frac{1}{2}\right)}=\varphi_{0}(x)^{(\ell)}, \quad n=0 \\
& \frac{1}{2} \frac{d \varphi_{n+1}(x)^{\left(\ell+\frac{1}{2}\right)}}{d x}+n \frac{d \varphi_{n-1}(x)^{\left(\ell+\frac{1}{2}\right)}}{d x}+\varphi_{n}(x)^{\left(\ell+\frac{1}{2}\right)}=0, \quad n=1,2, \ldots, N,
\end{aligned}
$$


where

$$
\varphi_{n}(x)^{\left(\ell+\frac{1}{2}\right)}=\frac{1}{\sqrt{\pi}} \int_{-\infty}^{\infty} H_{n}(\mu) \psi(x, \mu)^{(\ell+1 / 2)} e^{-\mu^{2}} d \mu
$$

with $H_{n}(\mu)$ denoting the $n$th order Hermite polynomials. Then the accelerated synthetic equations are defined as

(30a) $\frac{d \varphi_{1}(x)^{(\ell+1)}}{d x}=0, \quad n=0$

(30b) $\frac{1}{2} \frac{d \varphi_{n+1}(x)^{(\ell+1)}}{d x}+n \frac{d \varphi_{n-1}(x)^{(\ell+1)}}{d x}+\varphi_{n}(x)^{(\ell+1)}=0, \quad n=1,2, \ldots, N-1$,

and

$$
N \frac{d \varphi_{N-1}(x)^{(\ell+1)}}{d x}+\varphi_{N}(x)^{(\ell+1)}=-\frac{1}{2} \frac{d \varphi_{N+1}(x)^{\left(\ell+\frac{1}{2}\right)}}{d x}, \quad n=N .
$$

It is noticed that all moments of $\psi(x, \mu)$, except the $N+1$ moment, are accelerated. The above set of equations is coupled with the transport equation (1) and they are solved in an iterative manner. More specifically the proposed accelerated DVM consists of the following steps:

1. Assume $\varphi_{0}(x)^{(\ell)}$ and compute $\psi(x, \mu)^{(\ell+1 / 2)}$ from $(1)$.

2. Estimate the $N+1$ moment of $\psi(x, \mu)$, which is not accelerated, from $(29)$.

3. Solve the system of differential equations $(30)$ and find $\varphi_{n}(x)^{(\ell+1)}$ for $n=$ $0,1, \ldots, N$.

4. Check for convergence between $\varphi_{0}(x)^{(\ell+1)}$ and $\varphi_{0}(x)^{(l)}$ and if necessary go back to step 1 and use the accelerated $\varphi_{0}(x)^{(\ell+1)}$ to compute the new $\psi(x, \mu)$.

Various acceleration schemes may be defined, depending upon the number of accelerated moments. In general when the first $N$ Hermite moments are accelerated, we name the corresponding acceleration algorithm the " $\mathrm{H}_{\mathrm{N}}$ acceleration scheme," consisting of the transport equation, the $N+1(0,1, \ldots, N)$ synthetic equations, and the integral expression for the nonaccelerated $N+1$ moment. It is obvious that the computational effort per iteration is increased. However, as it is proved in section 4 theoretically and in section 5 experimentally, the number of required iterations for convergence is significantly reduced, and the overall computational scheme becomes very efficient.

We now turn our attention to the formulation of the proposed acceleration algorithms in the case of two-dimensional Cartesian geometry. The procedure is demonstrated by applying an $\mathrm{H}_{1}$ acceleration scheme to the linearized BGK equation in $x-y$ geometry. To carry this out, $(20)$ is multiplied first by $\frac{1}{\Pi} e^{-\mu^{2}-\eta^{2}}$ and then successively by $H_{0}(\mu) H_{0}(\eta), H_{1}(\mu) H_{0}(\eta)$, and $H_{0}(\mu) H_{1}(\eta)$. The resulting equations are integrated over $\mu$ and $\eta$ yielding the following set of synthetic equations:

$$
\begin{aligned}
& \frac{d \varphi_{10}^{(\ell+1)}}{d x}+\frac{d \varphi_{01}^{(\ell+1)}}{d y}=0 \\
& \frac{d \varphi_{00}^{(\ell+1)}}{d x}+\varphi_{10}^{(\ell+1)}=-\frac{1}{2} \frac{d \varphi_{20}^{\left(\ell+\frac{1}{2}\right)}}{d x}-\frac{1}{2} \frac{d \varphi_{11}^{\left(\ell+\frac{1}{2}\right)}}{d y},
\end{aligned}
$$

and

$$
\frac{d \varphi_{00}^{(\ell+1)}}{d y}+\varphi_{01}^{(\ell+1)}=-\frac{1}{2} \frac{d \varphi_{02}^{\left(\ell+\frac{1}{2}\right)}}{d y}-\frac{1}{2} \frac{d \varphi_{11}^{\left(\ell+\frac{1}{2}\right)}}{d x}
$$


where

$$
\varphi_{m n}^{\left(\ell+\frac{1}{2}\right)}(x, y)=\frac{1}{\pi} \int_{-\infty}^{\infty} \int_{-\infty}^{\infty} \psi^{\left(\ell+\frac{1}{2}\right)}(x, y, \mu, \eta) H_{m}(\mu) H_{n}(\eta) e^{-\mu^{2}-\eta^{2}} d \mu d \eta .
$$

The iterative procedure applied to the accelerated $\mathrm{H}_{1}$ algorithm, consisting of (20), (31), and (32), is identical to the one-dimensional case. This two-dimensional $\mathrm{H}_{1}$ acceleration scheme is applied in section 5 to solve the gas flow problems.

3.2. The $\mathbf{S}$ model kinetic equation. The iterative acceleration scheme for the solution of the more advanced S model kinetic equations may be formulated in a similar manner. Some modifications are required, however, due to the special form of the two-coupled transport equations. First (11) is rewritten in the revised form

$$
\begin{aligned}
& \mu \frac{\partial \psi_{1}^{\left(\ell+\frac{1}{2}\right)}}{\partial x}+\psi_{1}(x, \mu)^{\left(\ell+\frac{1}{2}\right)} \\
& \quad=f_{0}(x)^{(\ell)}+\frac{2}{15}\left(\mu^{2}-\frac{1}{2}\right)\left[\frac{1}{4} f_{2}(x)^{(\ell)}-2 f_{0}(x)^{(\ell)}+2 h_{0}(x)^{(\ell)}\right]
\end{aligned}
$$

and

$$
\begin{aligned}
& \mu \frac{\partial \psi_{2}^{\left(\ell+\frac{1}{2}\right)}}{\partial x}+\psi_{2}(x, \mu)^{\left(\ell+\frac{1}{2}\right)} \\
& \quad=f_{0}(x)^{(\ell)}+\frac{2}{15}\left(\mu^{2}+\frac{1}{2}\right)\left[\frac{1}{4} f_{2}(x)^{(\ell)}-2 f_{0}(x)^{(\ell)}+2 h_{0}(x)^{(\ell)}\right]
\end{aligned}
$$

where

$$
f_{n}(x)^{(\ell+1)}=\frac{1}{\sqrt{\pi}} \int_{-\infty}^{\infty} H_{n}(\mu) \psi_{1}(x, \mu)^{\left(\ell+\frac{1}{2}\right)} e^{-\mu^{2}} d \mu
$$

and

$$
h_{n}(x)^{(\ell+1)}=\frac{1}{\sqrt{\pi}} \int_{-\infty}^{\infty} H_{n}(\mu) \psi_{2}(x, \mu)^{\left(\ell+\frac{1}{2}\right)} e^{-\mu^{2}} d \mu .
$$

Next the two coupled kinetic equations (33) are first multiplied by the exponential term $\frac{1}{\sqrt{\pi}} e^{-\mu^{2}}$ and then the $N+1$ Hermitian moments of the resulting equations are taken. The coupling between the solution of the transport equations and the accelerated synthetic equations is achieved through the two (in this case) $N+1$ moments, which are not accelerated. Since the general $\mathrm{H}_{\mathrm{N}}$ acceleration algorithm has been described in the previous section for the BGK model extensively and due to the fact that the involved mathematical manipulation with the $\mathrm{S}$ model equations is considerably increased, we demonstrate only the formulation of the $\mathrm{H}_{1}$ scheme. Equations (33) are multiplied successively by $\frac{1}{\sqrt{\pi}} H_{0}(\mu) e^{-\mu^{2}}$ and $\frac{1}{\sqrt{\pi}} H_{1}(\mu) e^{-\mu^{2}}$ and the resulting four equations are integrated over $\mu$ to yield

$$
\begin{aligned}
& \frac{1}{2} \frac{d f_{1}(x)^{\left(\ell+\frac{1}{2}\right)}}{d x}+f_{0}(x)^{\left(\ell+\frac{1}{2}\right)}=f_{0}(x)^{(\ell)}, \\
& \frac{1}{2} \frac{d h_{1}(x)^{\left(\ell+\frac{1}{2}\right)}}{d x}+h_{0}(x)^{\left(\ell+\frac{1}{2}\right)}=\frac{11}{15} f_{0}(x)^{(\ell)}+\frac{1}{30} f_{2}(x)^{(\ell)}+\frac{4}{15} h_{0}(x)^{(\ell)},
\end{aligned}
$$


and

$$
\begin{aligned}
& \frac{d f_{0}(x)^{\left(\ell+\frac{1}{2}\right)}}{d x}+f_{1}(x)^{\left(\ell+\frac{1}{2}\right)}=-\frac{1}{2} \frac{d f_{2}(x)^{\left(\ell+\frac{1}{2}\right)}}{d x}, \\
& \frac{d h_{0}(x)^{\left(\ell+\frac{1}{2}\right)}}{d x}+h_{1}(x)^{\left(\ell+\frac{1}{2}\right)}=-\frac{1}{2} \frac{d h_{2}(x)^{\left(\ell+\frac{1}{2}\right)}}{d x},
\end{aligned}
$$

respectively. Then the synthetic equations are

$$
\begin{aligned}
& \frac{d f_{1}(x)^{(\ell+1)}}{d x}=0, \\
& \frac{1}{2} \frac{d h_{1}(x)^{(\ell+1)}}{d x}+\frac{11}{15}\left[h_{0}(x)^{(\ell+1)}-f_{0}(x)^{(\ell+1)}\right]=\frac{1}{30} f_{2}(x)^{\left(\ell+\frac{1}{2}\right)}, \\
& \frac{d f_{0}(x)^{(\ell+1)}}{d x}+f_{1}(x)^{(\ell+1)}=-\frac{1}{2} \frac{d f_{2}(x)^{\left(\ell+\frac{1}{2}\right)}}{d x}, \\
& \frac{d h_{0}(x)^{(\ell+1)}}{d x}+h_{1}(x)^{(\ell+1)}=-\frac{1}{2} \frac{d h_{2}(x)^{\left(\ell+\frac{1}{2}\right)}}{d x},
\end{aligned}
$$

The accelerated DVM consists of the two-coupled kinetic equations (33) and the synthetic equations (36), while the two second order moments of $\psi_{1}(x, \mu)$ and $\psi_{2}(x, \mu)$, which are not accelerated, are defined by

$$
f_{2}(x)^{\left(\ell+\frac{1}{2}\right)}=\frac{1}{\sqrt{\pi}} \int_{-\infty}^{\infty} H_{2}(\mu) \psi_{1}(x, \mu)^{\left(\ell+\frac{1}{2}\right)} e^{-\mu^{2}} d \mu
$$

and

$$
h_{2}(x)^{\left(\ell+\frac{1}{2}\right)}=\frac{1}{\sqrt{\pi}} \int_{-\infty}^{\infty} H_{2}(\mu) \psi_{2}(x, \mu)^{\left(\ell+\frac{1}{2}\right)} e^{-\mu^{2}} d \mu,
$$

respectively. It is seen that the procedure is quite similar as before, although the computational effort is doubled since twice as many synthetic equations are solved per iteration. The overall efficiency, however, of the accelerated scheme compared to the typical DVM is again significantly increased. The procedure can be extended in a straightforward manner to various $\mathrm{H}_{\mathrm{N}}$ acceleration schemes.

4. Theoretical convergence rates of acceleration schemes for the BGK model. Next the spectral radius of the proposed acceleration schemes is studied for the case of the BGK model. The algorithm is amenable to Fourier analysis, and we operate in a similar manner as for the typical iterative schemes studied in section 2 . Subtracting (1), (30), and (29) for successive values of $\ell$ we obtain transport equation (5) and the correction synthetic equations

$$
\begin{aligned}
& \frac{d \Phi_{1}(x)^{(\ell+1)}}{d x}=0, \quad n=0 \\
& \frac{1}{2} \frac{d \Phi_{n+1}(x)^{(\ell+1)}}{d x}+n \frac{d \Phi_{n-1}(x)^{(\ell+1)}}{d x}+\Phi_{n}(x)^{(\ell+1)}=0, \quad n=1,2, \ldots, N-1
\end{aligned}
$$

and

$$
N \frac{d \Phi_{N-1}(x)^{(\ell+1)}}{d x}+\Phi_{N}(x)^{(\ell+1)}=-\frac{1}{2} \frac{d \Phi_{N+1}(x)^{\left(\ell+\frac{1}{2}\right)}}{d x}, \quad n=N
$$


while the $N+1$ moment, which is not accelerated is defined by

$$
\Phi_{N+1}(x)^{\left(\ell+\frac{1}{2}\right)}=\frac{1}{\sqrt{\pi}} \int_{-\infty}^{\infty} H_{N+1}(\mu) Y(x, \mu)^{\left(\ell+\frac{1}{2}\right)} e^{-\mu^{2}} d \mu .
$$

The single Fourier error mode

$$
\Phi_{n}(x)^{(\ell+1)}=\omega \Phi_{n}(x)^{(\ell)}=\omega^{\ell+1} \alpha_{n} e^{i \lambda x}, \quad n=0,1,2, \ldots, N,
$$

along with (39) are introduced into (38), to yield after some algebraic manipulation the vector equation

$$
\omega D a=-i \lambda V G_{N+1},
$$

where $a^{T}=\left(a_{0}, a_{1}, \ldots, a_{N}\right)$ and $V^{T}=(0,0, \ldots, 0,1)$ are two constant vectors, $D$ is a tridiagonal matrix given by

$$
D(\lambda)=\left\{\begin{array}{cccccc}
0 & \frac{i \lambda}{2} & 0 & & & \\
i \lambda & 1 & \frac{i \lambda}{2} & & & \\
& \cdot & \cdot & \cdot & & \\
& & n i \lambda & 1 & \frac{i \lambda}{2} & \\
& & \cdot & \cdot & \cdot & \\
& & & 0 & N i \lambda & 1
\end{array}\right\}
$$

with $n=0,1,2, \ldots, N$ and $G_{N+1}$ is the scalar quantity

$$
G_{N+1}=\frac{1}{2 \sqrt{\pi}} \int_{-\infty}^{\infty} H_{N+1}(\mu) y(\mu) e^{-\mu^{2}} d \mu .
$$

Introducing (9) for the eigenfunction $y(\mu),(43)$ is written in the more convenient form

$$
G_{N+1}=F \cdot \alpha,
$$

where $\mathrm{F}$ is a row vector with components

$$
F_{0}(\lambda)=\frac{1}{2 \sqrt{\pi}} \int_{-\infty}^{\infty} H_{N+1}(x) \mu \frac{e^{-\mu^{2}}}{1+i \lambda \mu} d \mu
$$

and

$$
F_{n}(\lambda)=0, \quad n=1,2, \ldots, N .
$$

Then (41) leads to an eigenvalue problem written as

$$
(A-\omega I) \alpha=0
$$

with

$$
A=-i \lambda D^{-1} V F \text {. }
$$

It is obvious that in this eigenvalue problem $\omega$ are the eigenvalues of $A$ and $a$ the corresponding eigenvectors. According to (46), however, $A$ is the product of a column 


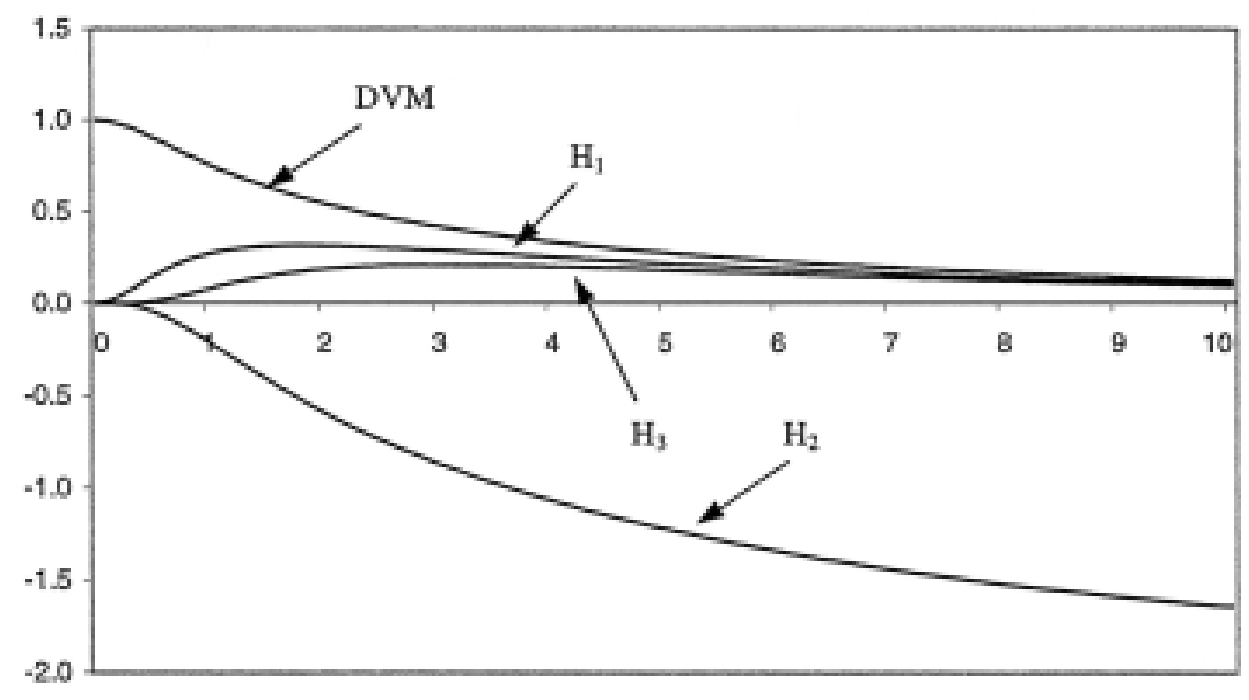

FIG. 2. Plots of $\omega$ versus $\lambda$ for various orders of $\mathrm{H}_{\mathrm{N}}$ acceleration and the typical DVM (BGK model).

vector times a row vector and because of that its rank is equal to unity. Hence we obtain one dominant eigenvalue, while the $N$ remaining eigenvalues are identically equal to zero. In fact, by multiplying both sides of the vector equation (45) by $F$, the following scalar equation is reduced:

$$
\omega=-i \lambda F D^{-1} V .
$$

Thus study of the one nonzero eigenvalue is sufficient to obtain the convergence rate of the acceleration scheme.

Applying this $\mathrm{H}_{\mathrm{N}}$ acceleration analysis by accelerating the first two, three, and four moments of the distribution function we find the explicit results

$$
\begin{aligned}
& \omega_{1}=\Lambda+\frac{2}{\lambda^{2}}(\Lambda-1), \\
& \omega_{2}=3 \Lambda+\frac{2}{\lambda^{2}}(\Lambda-1)-2,
\end{aligned}
$$

and

$$
\omega_{3}=\frac{1}{2+3 \lambda^{2}}\left\{3 \lambda^{2} \Lambda-4(1-\Lambda)\left(3+\frac{1}{\lambda^{2}}\right)+2\right\} .
$$

The above closed form expressions are the eigenvalues of the acceleration algorithms described by (1), (29), and (30), with $N$ equal to one, two, and three, respectively, while $\Lambda$ is the integral defined in (10) and denotes the convergence rate $\omega_{\mathrm{DVM}}$ of the typical DVM applied to the BGK equation.

Equations (48) are solved numerically and the results are plotted in Figure 2, where the plot of $\omega_{D V M}$ is also included for comparison. Some theoretical insight into the behavior of $\omega$ as a function of $\lambda$ is provided for the three acceleration schemes and the DVM. Table 1 contains the spectral radius for the typical DVM and each of the three iteration acceleration schemes $\left(\mathrm{H}_{1}, \mathrm{H}_{2}\right.$, and $\left.\mathrm{H}_{3}\right)$ under consideration. 
TABLE 1

Spectral radius of the typical DVM and the synthetic acceleration methods $\mathrm{H}_{1}, \mathrm{H}_{2}$, and $\mathrm{H}_{3}$ for the BGK model.

\begin{tabular}{|l|l|l|l|l|}
\hline Method & DVM & $\mathrm{H}_{1}$ & $\mathrm{H}_{2}$ & $\mathrm{H}_{3}$ \\
\hline Spectral radius & 1.00 & 0.320 & 2.00 & 0.211 \\
\hline
\end{tabular}

The well-known inefficiency of the $\mathrm{H}_{2}$ scheme is easily observed [21]. The $\mathrm{H}_{1}$ acceleration scheme seems to be the most efficient acceleration scheme. Two $\mathrm{H}_{1}$ sweeps will reduce the error by a factor of about ten, while a single $\mathrm{H}_{3}$ sweep requires at least the same computational time and will reduce the error only by a factor of about five.

The convergence rate of the $\mathrm{H}_{1}$ acceleration scheme for the two-dimensional BGK equation described by (20), (31), and (32) is studied next. Equations (31) are subtracted for successive iterates and the ansatz

$$
\begin{aligned}
& \Phi_{00}(x)^{(\ell+1)}=\omega \Phi_{00}(x)^{(\ell)}=\omega^{(\ell+1)} \alpha_{00} e^{i \lambda x}, \\
& \Phi_{10}(x)^{(\ell+1)}=\omega \Phi_{10}(x)^{(\ell)}=\omega^{(\ell+1)} \alpha_{10} e^{i \lambda x},
\end{aligned}
$$

and

$$
\Phi_{01}(x)^{(\ell+1)}=\omega \Phi_{01}(x)^{(\ell)}=\omega^{(\ell+1)} \alpha_{01} e^{i \lambda x}
$$

is substituted into the resulting equations to yield

$$
\begin{aligned}
& \omega\left(i \lambda \theta_{x} a_{10}+\lambda \theta_{y} a_{01}\right)=0 \\
& \omega\left(i \lambda \theta_{x} a_{00}+a_{10}\right)=-\frac{1}{2} i\left(\lambda \theta_{x} G_{20}+\lambda \theta_{y} G_{11}\right),
\end{aligned}
$$

and

$$
\omega\left(\lambda \theta_{y} a_{00}+a_{01}\right)=-\frac{1}{2} i\left(\lambda \theta_{y} G_{02}+\lambda \theta_{x} G_{11}\right),
$$

where

$$
G_{m n}(x, y)=\frac{1}{\pi} \int_{-\infty}^{\infty} \int_{-\infty}^{\infty} y(\mu, \eta) H_{m}(\mu) H_{n}(\eta) e^{-\mu^{2}-\eta^{2}} d \mu d \eta
$$

Thus in general there are three eigenvalues for each $\lambda$. Introducing (26) for the eigenfunction $y(\mu, \eta)$ in (50) and after some routine manipulation we find $\omega_{1}=\omega_{2}=0$ and the dominant eigenvalue

$$
\omega_{3}=\frac{1}{\pi} \int_{-\infty}^{\infty} \int_{-\infty}^{\infty}\left[1-2\left(\mu \theta_{x}+\eta \theta_{y}\right)^{2}\right] y(\mu, \eta) e^{-\mu^{2}-\eta^{2}} d \mu d \eta
$$

The double integral in the explicit expression for $\omega_{3}$ can be evaluated analytically to yield (48a), which has been obtained for the one-dimensional $\mathrm{H}_{1}$ acceleration. The point to be stressed here is that the $\mathrm{H}_{1}$ scheme results in the same closed form expression for the dominant eigenvalue for both the one- and two-dimensional problems. The same conclusion has been found earlier in section 2 for the DVM. Similar results can be obtained for any $\mathrm{H}_{\mathrm{N}}$ acceleration algorithm. 
5. Gaseous flows in rectangular microchannels. In this section the theoretical results obtained earlier are confirmed experimentally. The flow of a gas in a rectangular microchannel caused by small pressure and temperature gradients in the axial direction is selected as a test case. Both the isothermal and nonisothermal flows implementing the BGK and S models, respectively, have been considered. They are described by two-dimensional transport equations similar to (20) and (21), by selecting the appropriate source terms. In the present work they are used as a test bed for the proposed acceleration scheme. Both problems have been solved recently by Sharipov [15] and Sharipov and Seleznev [16], and recent results exist for the whole range of the Kn number and various ratios of width over height, based on the typical DVM. Here the two problems are solved again using the DVM and also the $\mathrm{H}_{1}$ acceleration algorithm. For the BGK model the formulation of the DVM is described by (20) and (21) and of the $\mathrm{H}_{1}$ scheme by (20), (31), and (32). For simplicity, purely diffused Maxwell boundary conditions [7] with no specular reflection are considered. A similar formulation is derived for the two-dimensional $\mathrm{S}$ model.

The experimental investigation of the effectiveness of the algorithm is based on the calculation of overall quantities, such as flow rates and heat fluxes, which are of main interest for engineering purposes. Our objective here is to find and compare the required number of iterations in order to achieve the same specified convergence criterion and accuracy for the two approaches. For that reason we decide not to tabulate explicitly the macroscopic quantities but only the required number of iterations for the typical and the acceleration iterative methods in terms of the convergence criteria. To achieve that, the analytic transport and synthetic equations have been discretized in space using the diamond-difference scheme [22], while a typical double Gauss-Hermite quadrature approximation has been applied for the velocity discretization. It is well known that consistent differencing between the transport and the synthetic equations is essential to avoid conditional instabilities in the acceleration schemes $[1,2,21]$. Exactly the same principal applies in the present formulation.

A first comparison between the DVM and the $\mathrm{H}_{1}$ acceleration scheme is shown for the BGK model in Figure 3. The required number of iterations to satisfy the convergence criterion in the computed results is plotted as a function of the convergence criterion. The results are presented by Knudsen numbers equal to 1, 0.1, and 0.01 and for the case of a square microchannel, with a set of 64 discrete velocities and 21 spatial nodes in each direction. It is seen that for $\mathrm{Kn}=1$ the number of required iterations is very small and there is no benefit in using the $\mathrm{H}_{1}$ scheme. For $\mathrm{Kn}=0.1$ and $\mathrm{Kn}=0.01$, however, the required number of iterations for the typical DVM is significantly increased, while it remains small for the $\mathrm{H}_{1}$ acceleration scheme. More specifically for a relative convergence criterion of about $10^{-4}$ the required iterations for the $\mathrm{H}_{1}$ scheme are reduced roughly by a factor of 10 for the case of $\mathrm{Kn}=0.1$ and by a factor of $10^{3}$ for the case of $\mathrm{Kn}=0.01$. It is also noticed that in these cases $(\mathrm{Kn}$ equal to 0.1 and 0.01 ) the required number of iterations for the acceleration scheme remains actually constant even when very strict convergence criteria are applied. The corresponding results in the case of the $\mathrm{S}$ model are shown in Figure 4 and they are very similar with those of Figure 3. This is an interesting fact indicating that the effectiveness of the acceleration algorithm is computationally independent of the complexity of the kinetic model.

In Figures 5 and 6 the required CPU time to satisfy the convergence criterion in the computed results is plotted in terms of the convergence criterion for the BGK and the $\mathrm{S}$ models, respectively. The Knudsen number is taken equal to 0.01 . It is seen that 

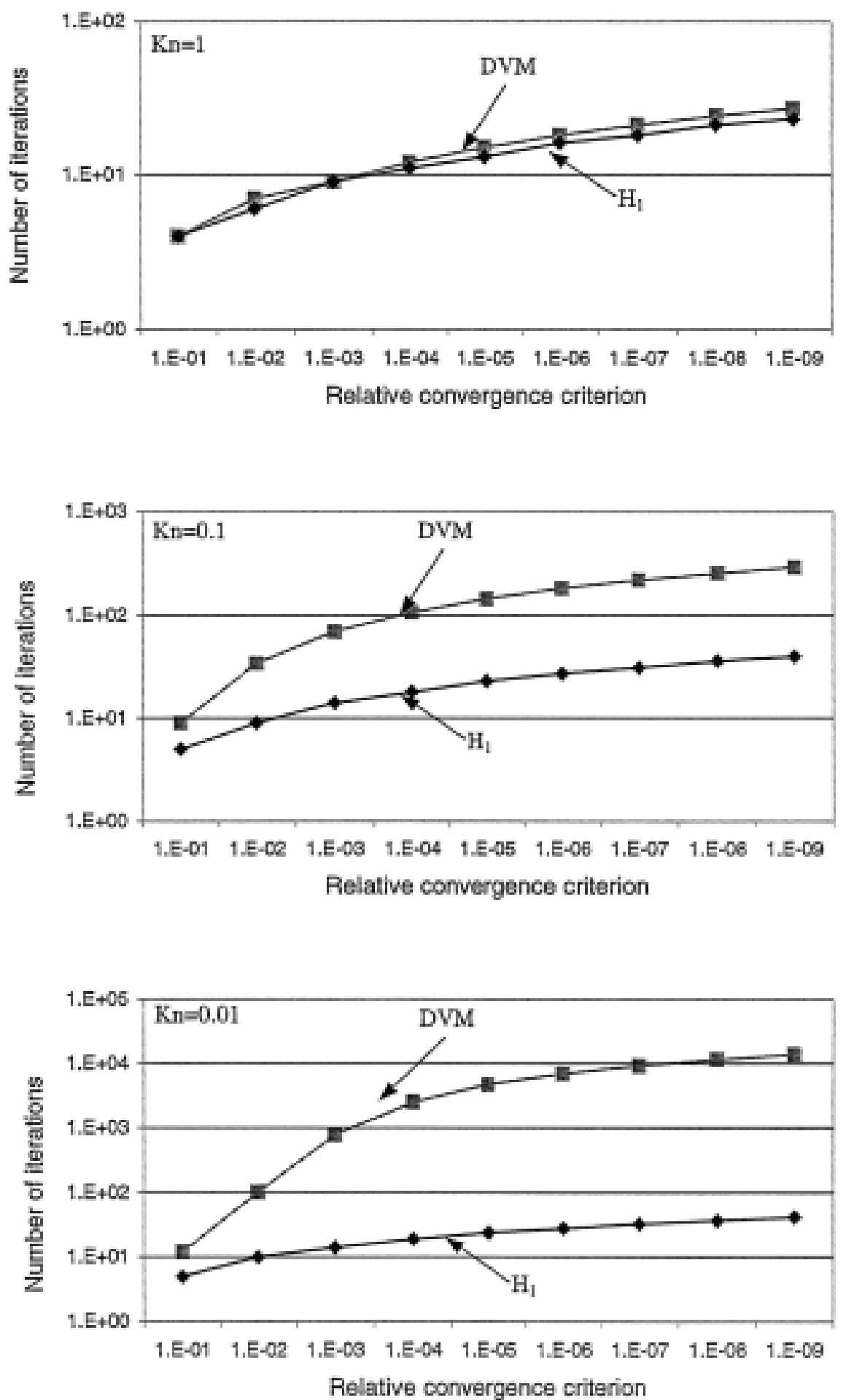

FIG. 3. Comparison between the DVM and the $\mathrm{H}_{1}$ acceleration method in terms of the required number of iterations to satisfy the convergence criterion for the BGK model and various Knudsen numbers. 

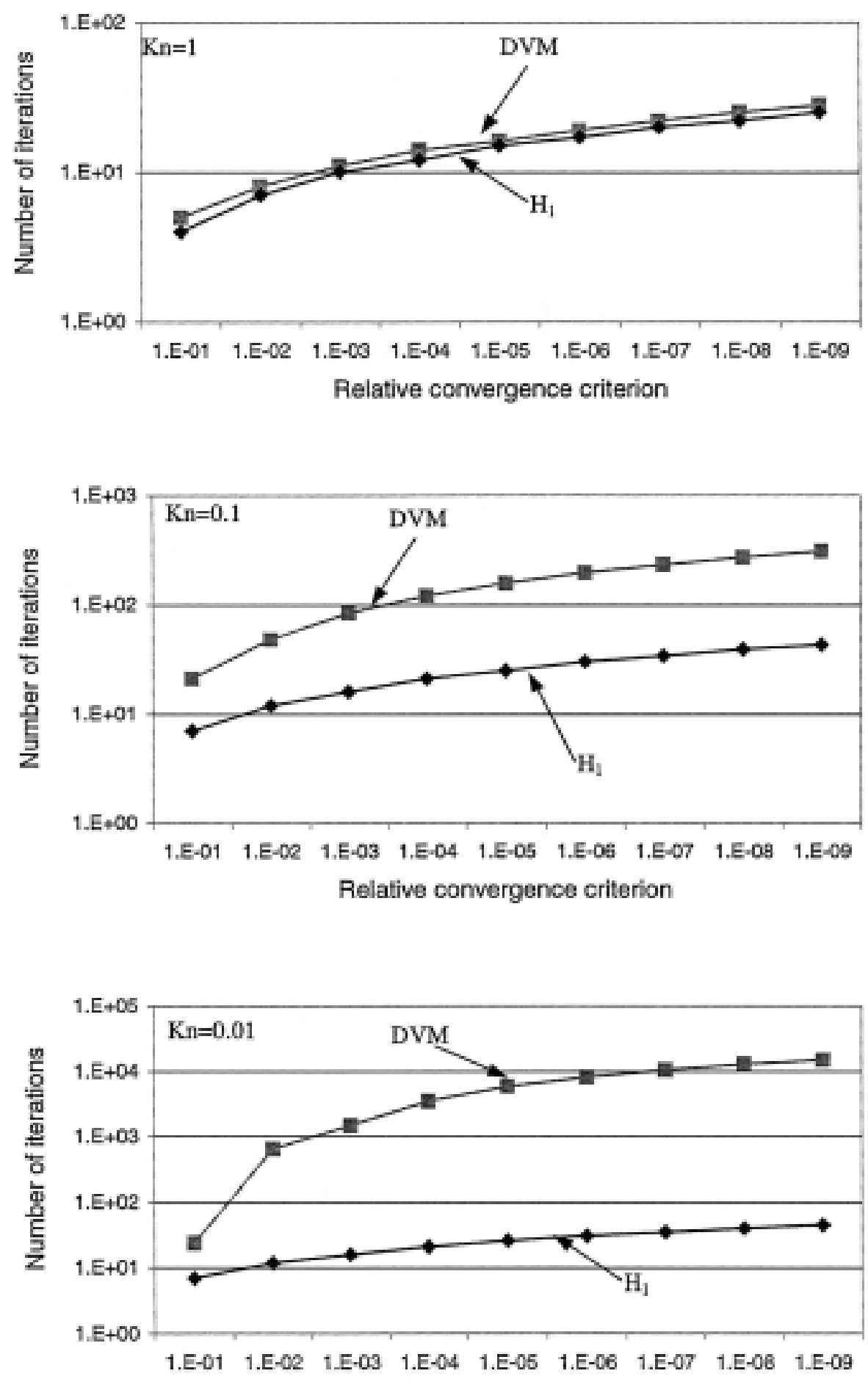

Relative convergence criterion

FIG. 4. Comparison between the DVM and the $\mathrm{H}_{1}$ acceleration method in terms of the required number of iterations to satisfy the convergence criterion for the $S$ model and various Knudsen numbers. 


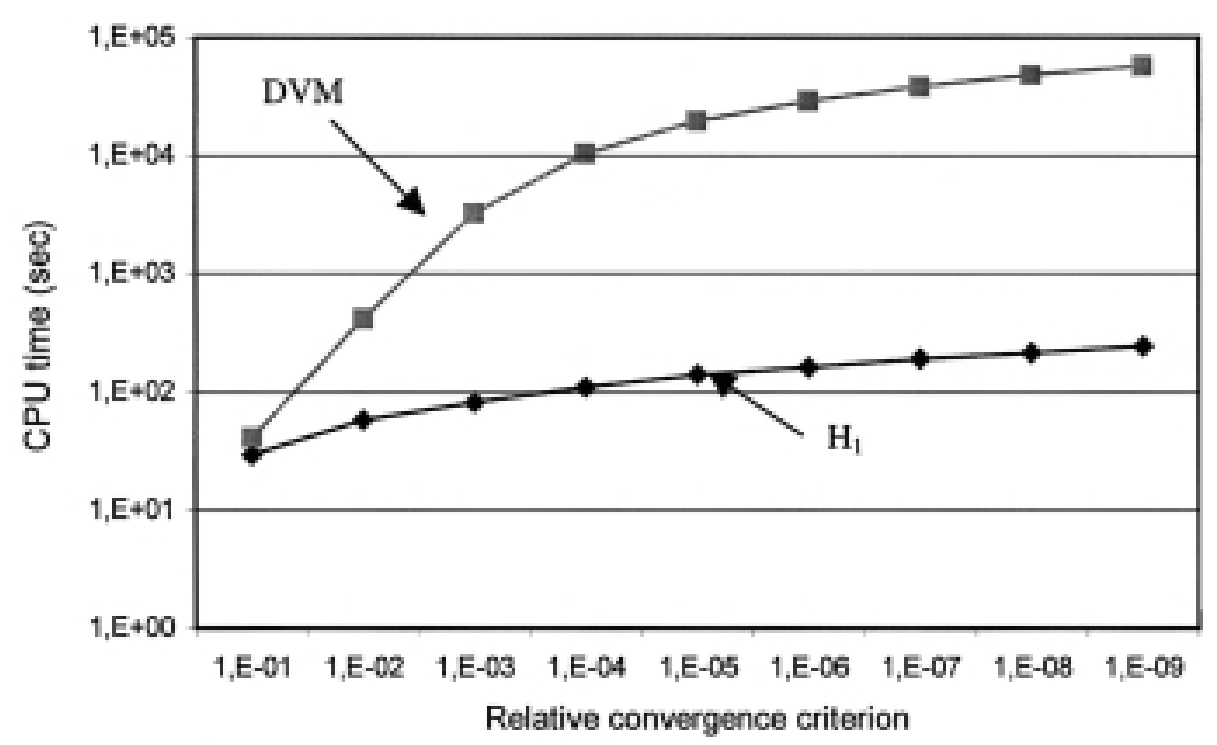

FIG. 5. Comparison between the DVM and the $\mathrm{H}_{1}$ acceleration method in terms of the required $C P U$ time to satisfy the convergence criterion for the BGK model and $K n=0.01$.

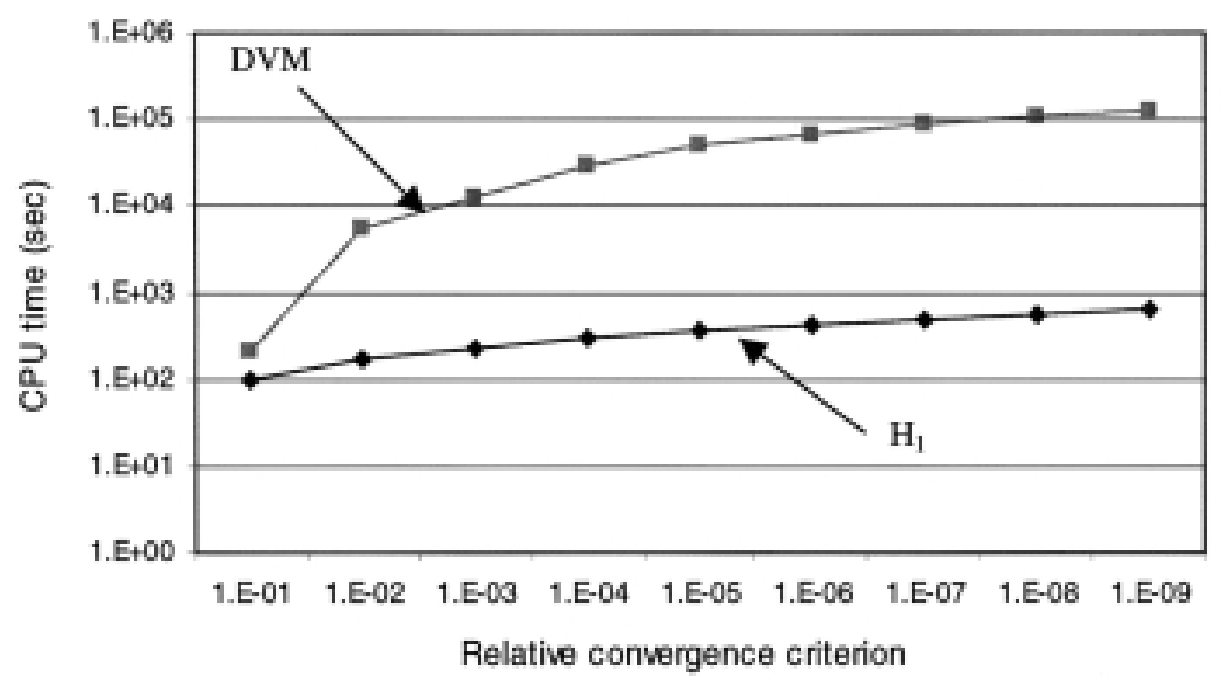

FiG. 6. Comparison between the DVM and the $\mathrm{H}_{1}$ acceleration method in terms of the required $C P U$ time to satisfy the convergence criterion for the $S$ model and $K n=0.01$.

the reduction in the overall computational time is of the same order of magnitude with the reduction in the required number of iterations. This is easily explained by the fact that the additional computational effort per iteration is insignificant compared to the computational gain due to the small number of iterations required. The results have been obtained on a $700 \mathrm{MHz}$, Pentium III.

All previous results are very encouraging, although no special treatment of the boundary conditions has been applied. In the particular model problem, that was not necessary since simple homogeneous Dirichlet conditions are used. When all moments 
of the distribution function at the boundaries are not known, then derivation of acceleration equations at the boundary nodes incorporating the boundary conditions is required. When this formulation is fully compatible with the acceleration formulation for the internal nodes [22], then the efficiency of the overall acceleration algorithm remains high. This is an interesting issue, which will be studied in later work.

Overall it is clearly seen that the number of required iterations is significantly reduced when the accelerated scheme is applied, and this improvement becomes more important for small Knudsen numbers. Even more in this case the typical DVM results suffer from accumulated round-off error due to the large number of iterations required, and thus more strict convergence criterions do not always ensure more accurate results. In general, when the $\mathrm{H}_{1}$ accelerated scheme is introduced we expect that not only the computational effort is reduced but also the accuracy of the results is improved. Finally, it is noticed that, as it is expected, in all cases tested the discretized synthetic acceleration scheme performs equally well or even better than the exact scheme, applied on the continuous form of the transport and synthetic equations.

6. Conclusions. A Fourier stability analysis is performed to evaluate the convergence rate of the classical DVM implemented on the BGK and S models in slab and $x-y$ geometry. The spectral radius is found to be equal to unity, which justifies the well-known slow convergence of the method. By taking Hermite moments of the transport equation a set of synthetic equations is obtained. These acceleration equations are solved, coupled with the transport equation, and the convergence rate of the iteration map is significantly improved. The $\mathrm{H}_{1}$ acceleration scheme seems to be the most efficient speed up algorithm, from an overall computational point of view, while the even order acceleration schemes perform badly. The theoretical results are justified experimentally, solving the gaseous flow problem through a rectangular microchannel. The fact that the proposed acceleration scheme performs equally well for the BGK and the more advanced S model make us optimistic for extending the present work to the linearized Boltzmann equation with synthetic kernels.

\section{REFERENCES}

[1] M. L. Adams and E. W. Larsen, Fast iterative methods for discrete-ordinates particle transport calculations, Progress in Nuclear Energy, 40 (2002), pp. 3-159.

[2] R. E. Alcouffe, E. W. Larsen, W. F. Miller, Jr., and B. R. Wienke, Computational efficiency of numerical methods for the multigroup, discrete-ordinates neutron transport equations: The slab geometry case, Nuc. Sci. Eng., 71 (1979), pp. 111-127.

[3] P. L. Bhatnagar, E. P. Gross, and M. Krook, A model for collision processes in gases, Phys. Rev., 94 (1954), pp. 511-515.

[4] J. Broadwell, Study of rarefied shear flow by the discrete velocity method, J. Fluid Mech., 19 (1964), pp. 401-414.

[5] V. Bobylev and C. Cercignani, Discrete velocity models without nonphysical invariants, J. Statist. Phys., 97 (1999), pp. 677-689.

[6] H. Cabannes, Couette flow for a gas with a discrete velocity distribution, J. Fluid Mech., 76 (1976), pp. 273-287.

[7] C. Cercignani, The Boltzmann Equation and its Applications, Springer-Verlag, New York, 1988.

[8] C. Cercignani and F. Sharipov, Gaseous mixture slit flow at intermediate Knudsen numbers, Phys. Fluids, A, 4 (1992), pp. 1283-1289.

[9] C. Cecignani and A. V. Bobylev, Discrete velocity models: The case of mixtures, Transport Theory Statist. Phys., 29 (2000), pp. 209-216.

[10] A. B. Huang, P. F. Huang, D. P. Giddens, and R. SRinivasan, High-speed leading edge problem, Phys. Fluids, 16 (1973), pp. 814-824.

[11] E. E. Lewis And W. F. Miller, JR., Computational Methods of Neutron Transport, Wiley, New York, 1984. 
[12] S. K. Loyalka AND K. A. Hickey, Kinetic theory of thermal transpiration and the mechanocaloric effect: Planar flow of a rigid sphere gas with arbitrary accommodation at the surface, J. Vac. Sci. Technol., A9 (1991), pp. 158-163.

[13] E. M. Shakhov, Method of Investigation of Rarefied Gas Flows, Nauka, Moscow, 1974 (in Russian).

[14] F. SHARIPOV, Rarefied gas flow through a long tube at any temperature ratio, J. Vac. Sci. Technol., A14 (1996), pp. 2627-2635.

[15] F. Sharipov, Non-isothermal rarefied gas flow through a slit, Phys. Fluids, 9 (1997), pp. 18041810.

[16] F. Sharipov and V. Seleznev, Data in internal rarefied gas flows, J. Phys. Chem. Ref. Data, 27 (1998), pp. 657-706.

[17] F. Sharipov And E. A. Subbotin, On optimization of the discrete velocity method used in rarefied gas dynamics, Z. Angew. Math. Phys., 44 (1993), pp. 572-577.

[18] F. Sharipov, Rarefied gas flow through a long rectangular channel, J. Vac. Sci. Technol. A, 17 (1999), pp. 3062-3066.

[19] F. SHARIPOV, Non-isothermal gas flow through rectangular microchannels, J. Micromech. Microeng., 9 (1999), pp. 394-401.

[20] D. Valougeorgis, Couette flow of a binary gas mixture, Phys. Fluids, 31 (1988), pp. 521-524.

[21] D. Valougeorgis, M. Williams, and E. W. Larsen, Stability analysis of the synthetic acceleration methods with anisotropic scattering, Nuc. Sci. Eng., 99 (1988), pp. 91-98.

[22] D. Valougeorgis, Boundary treatment of the diffusion synthetic acceleration method for fixedsource discrete-ordinates problems in $x$-y geometry, Nuc. Sci. Eng., 100 (1988), pp. 142-148.

[23] M. Wakabayashi, T. Ohwada, And F. Golse, Numerical analysis of the shear and thermal creep flows of a rarefied gas over the plane wall of a Maxwell type boundary on the basis of the linearized Boltzmann equation for hard-sphere molecules, Eur. J. Mech. B Fluids, 15 (1996), pp. 175-202. 